\title{
A Review on Various Barcode Generation Techniques
}

\author{
Ninad Bandsode ${ }^{1}$, Abhishek Mukhedkar ${ }^{2}$, Tousif Tamboli ${ }^{3}$, Dhanshree Holkar ${ }^{4}$, Prof. Amit Zore \\ UG Student, Dhole Patil College of Engineering, Wagholi, Savitribai Phule Pune University 1, 2, 3, 4 \\ Professor, Dhole Patil College of Engineering, Wagholi, Savitribai Phule Pune University ${ }^{5}$
}

\begin{abstract}
As the secure data transmission has become a very important issue these days, various barcoding techniques have given a lot of relief in encoding the data into barcodes. The various barcodes like 1D Barcode, Line barcode, PDF457, QR Code, which have various data encoding techniques. But the major drawback of the existing barcodes is that the amount of data that can be encoded into barcodes is very small due to which becomes very difficult to encode larger data into these barcodes. So the paper makes a study about the existing barcoding techniques and thereby get a clear picture about what can be done for encoding the large amount of data. The comparative study of the existing systems is analysed in this paper.
\end{abstract}

Keywords: Barcoding techniques, data encoding, 2d Barcode, data transmission, security.

\section{INTRODUCTION}

This In facilitating numerous identification processes Barcodes have played a great role since their invention in 1952 [1]. In fact for machine readable digital data storing on product packages or paper, barcode is a cost-effective and simple method. Even faster data transfer as pressing needs and there have been many improvements with high reliability have emerged on the original barcode design that were made.

For these cost-effective codes as well as their application opened a new front by invention of HC2D barcodes in scenarios like storing contact information which transfer more complex data, URLs among other things, there have become increasingly popular in which QR codes [2]. There can be found the performance of camera phone applications in the comparison of 2D barcode in [3]. In matrix barcode development there have been dedicated much of the efforts on a piece of paper to barcodes displayed they are normally used as that is the way. That replacement of the paper with LCD One could contemplate with tablets and e-Book readers replacement with the books for broader applications of 2D barcodes may open another promising front as a mean of data transfer. Moreover for the eventual streams of data transfer, the LCD may display time-varying barcodes unlike the static paper to the receiving electronic device(s).

Through a series of HC2D barcode there transfer the data between two cell phones is studied, for state of the art mobile devices which achieving bit rates of under $10 \mathrm{kbps}$. With bit- rates of more than $14 \mathrm{Mbps}$ later for transmission and reception, a digital camera and a computer monitor are used. Over distances of up to 4 meters which achieved in docked transmitter and receiver conditions. However, when the distance is increased to 14 meters this rate drops to just over 2 Mbps.
Using a more effective coding and modulation scheme there is achieved the superior performance of the later implementation for mitigation of pixel and image blur to pixel light leakage. To modulate LCD pixels there used the inverse Fourier transform (IFT) of data like OFDM. The performance of QR decoders reduce by image light and blur leakage greatly. Furthermore to known portions of the decoded data there is confined their performance degradation.

Based on data region as in [5] on non-uniform error probability for adaptive error correction may be used coding this prior knowledge. In design as well as implementation of LCD-Camera based communication systems there is an increasing interest as indicated in [6][8]. For this type of innovative communications medium in determining optimal demodulation and modulation schemes this would require additional investigations.

For transferring data, there uses orthogonal frequency subcarriers by the OFDM modulation and image blur can confine, to high frequency components which is essentially a low pass filter are transmitted intact. To detect the data bits correctly this method requires high phase coherency. Through additional modifications this idea extends by the current study during the capture of a single frame in a way on the modulation scheme for mitigating LCD-camera relative movements, on the captured images which results in distortion of motion blur. As would be detailed later severely, the Quadrature Phase Shift Keying (QPSK) performance modulated signals of OFDM degrade due to this kind of distortion. From a single image which is shown on an LCD one should consider extracting maximum data to maximize data transmission rate and then at which consecutive frames will be the rate decoded increase. 
Vol. 5, Issue 12, December 2016

\section{LITERATURE SURVEY}

A. QR Code Detection in Arbitrarily Acquired Images Through creation of links between Internet resources and physical objects, there enable rich context interaction by applications of Quick Response (QR) codes. There are this kind of barcode applications are not common in spite of the widespread use for people and robots which are visually impaired because during image acquisition that the symbol is properly by existing decoders framed are assumed.
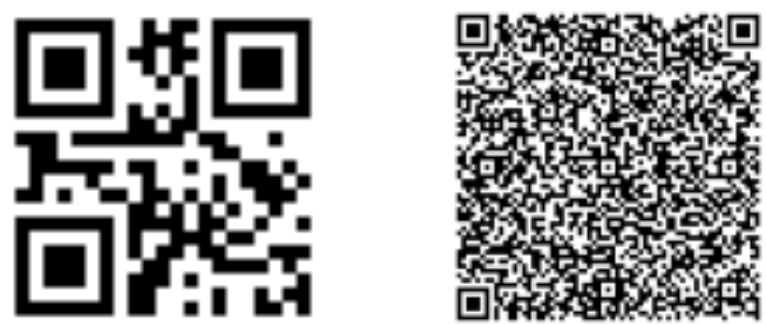

Figure 1. Different versions of QR code

To perform accurate detection of $\mathrm{QR}$ code symbols a two-stage component-based approach proposed by this work in arbitrarily acquired images.

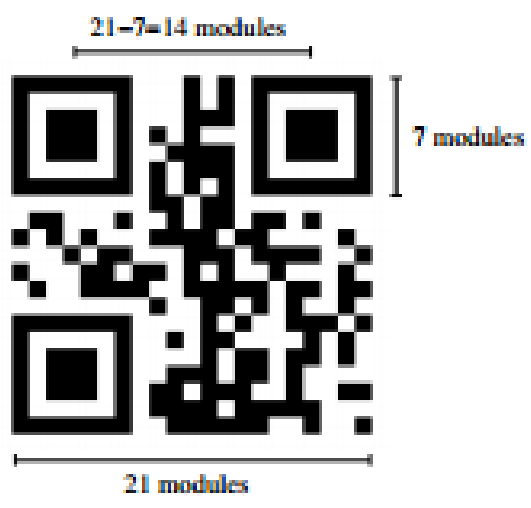

Figure 2. 2D QR code

With the help of the framework which detect the objects proposed by Viola-Jones to detect parts of the symbol is trained, a cascade classifier in the first stage. In the second stage, in order to evaluate the detected patterns are aggregated if they are spatially arranged with the components of a QR code symbol in a way that is geometrically consistent. There was performed an extensive study of both stages parameter variation and in terms of computational efficiency, recall and precision the results were analyzed. With precision of 76: $8 \%$ there achieved average recall of $91: 7 \%$ by the proposed $\mathrm{QR}$ code detector while at $22 \mathrm{fps}$ being capable of processing a 640 by 480 pixels video stream. For realtime applications implementation these results support which in mobile hardware assist visually impaired people and robots, through QR codes in multiple medium to the wealth of information available allowing them to have access.
B. 1D bar code reading on camera phones

In this paper, using a NOKIA 7650 , VGA camera phone on the bar code reading algorithms we present the research effort. From poor-quality images to extract bar code characters knowledge-based bar code segmentation and a wavelet-based bar code region location scheme is applied. For the recognition engine there are input all the characters which are segmented bar code, and the bar code character string as the final recognition result with the smallest total distance is output of the bar code based on the recognition distance. For optimizing the class reference vectors and a feature extraction matrix in order to train an efficient recognition engine, there is designed the modified Generalized Learning Vector Quantization (GLVQ) method. By NOKIA 7650 from more than 1000 bar code images captured, 584 samples segmented are involved in the training process.

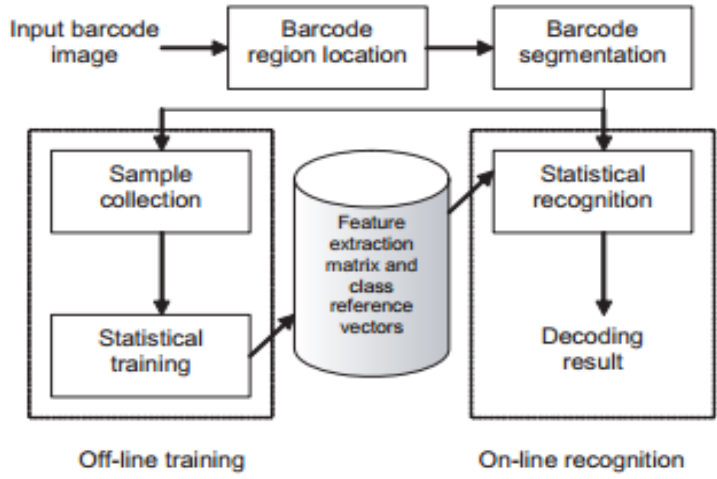

Figure 3. Diagram of the bar code decoding system

By the same phone the testing on 292 bar code images taken, there reaches $85.62 \%$ the entire bar code set of the correct recognition rate.

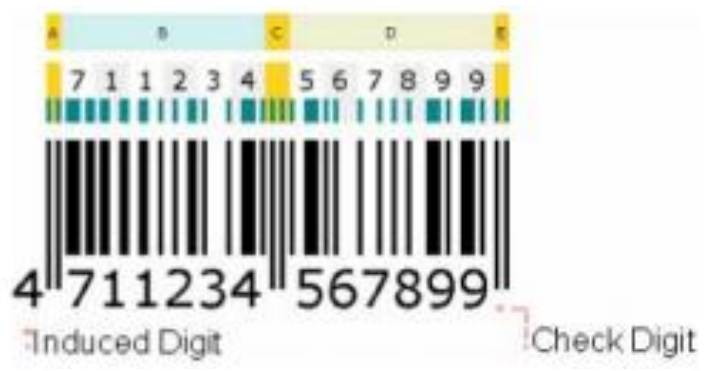

Figure 4. 1D barcode

C. Detecting and Decoding Algorithm for 2D Barcode

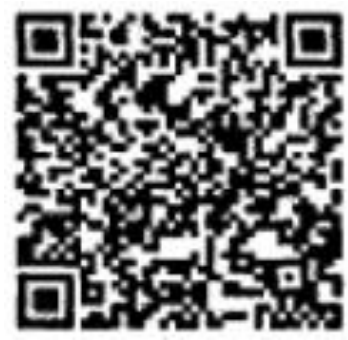

Figure 5. 2D barcode 
Vol. 5, Issue 12, December 2016

There can be classified the 2D Barcode mainly in two types which are matrix 2D barcode stacked 2Dbarcode. The structure of types of 2D barcode is discuss in this paper in brief. The flowchart of detecting 2D barcode propose this paper and the 2D barcode also decoding.

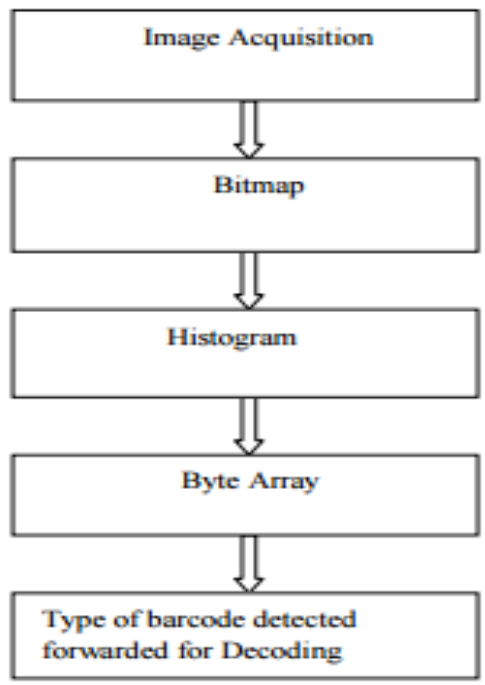

Figure 6. Detecting 2D barcode

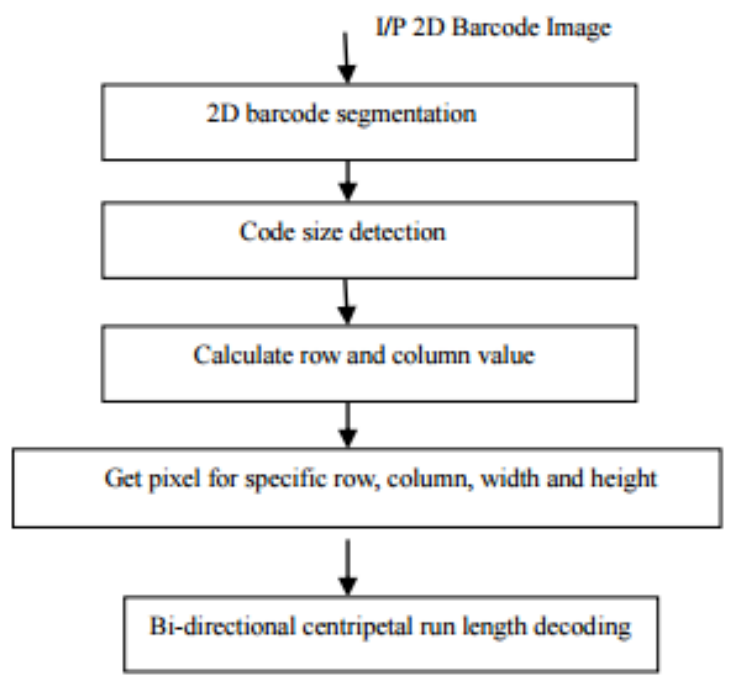

Figure 7. Decoding 2D barcode

D. Linear barcode scanning system based on dynamic template Matching for oof blurred images

In the spatial domain the proposed system works entirely and from severe OOF blur contain the low-resolution images is capable of reading Linear Barcodes. Under the perspective of deformed Binary waveform classification analysis, this paper treats linear barcode scanning. At any specific blur level for characterizing the relationship between the waveform of blurred barcode and value of its corresponding symbol a directed graphical model is designed. For enabling real-time decoding, retrieve the optimal state sequence there is designed a Dynamic programming-based inference algorithm on mobile devices of limited processing power.

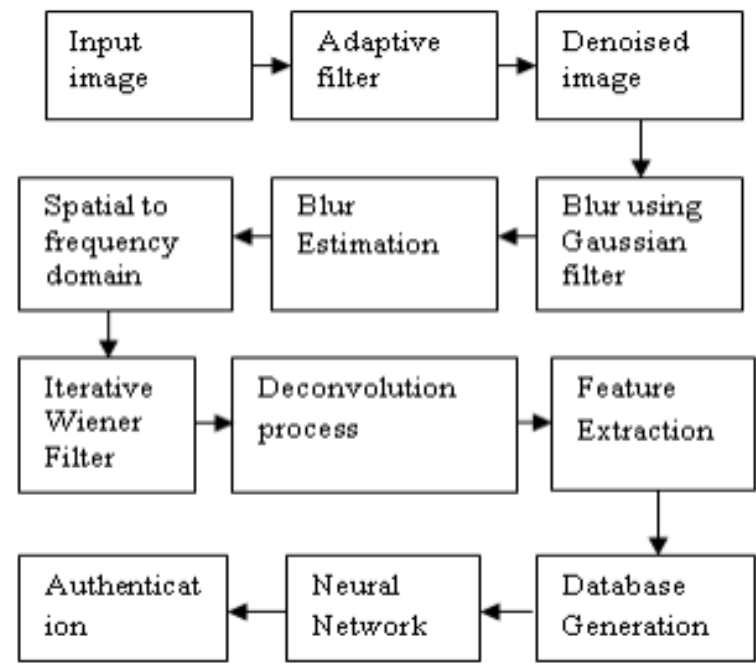

Figure 8. Dynamic template matching

E. Two-Dimensional Barcodes for Mobile Phones

For a high data density barcode there are several potential applications by mobile phones, that can be easily decoded and photographed, but currently no such symbology exists. As a result, for exploiting the camera phone channel's low-pass characteristic a new barcode was designed and with mobile phones as a means of facilitating wireless optical communication is presented. With encoding done in the Discrete Cosine Transform domain a channel model was established and subsequent simulation results led to the design of a colour barcode.
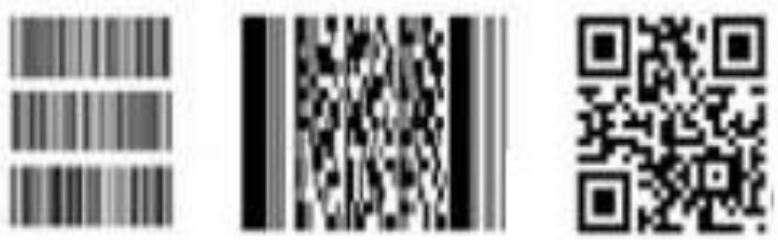

Figure 9. 2D barcodes

There enhance performance by a water-filling process and while for rotational and size invariance a noise-shaping algorithm, a new fast acquisition method allows. According to spatial frequency with a rate varying an outer Accumulate-Repeat-Accumulate code is employed, by an inner Reed Muller code which followed.

To various impediments imposed by camera phones for the leading hasproven and symbology robust there is 3.5 times greater the final barcode data-density.

F. QR Inception: Barcode-in-Barcode Attacks

In this paper, when especially crafted barcodes conform based on deliberately caused ambiguities we present novel attacks to multiple standards. Which standard the decoder locks on decides by implementation details. This way, with different phones or apps two users scanning the same barcode which will receive different content. For multiple problems related to security this potentially opens way. 


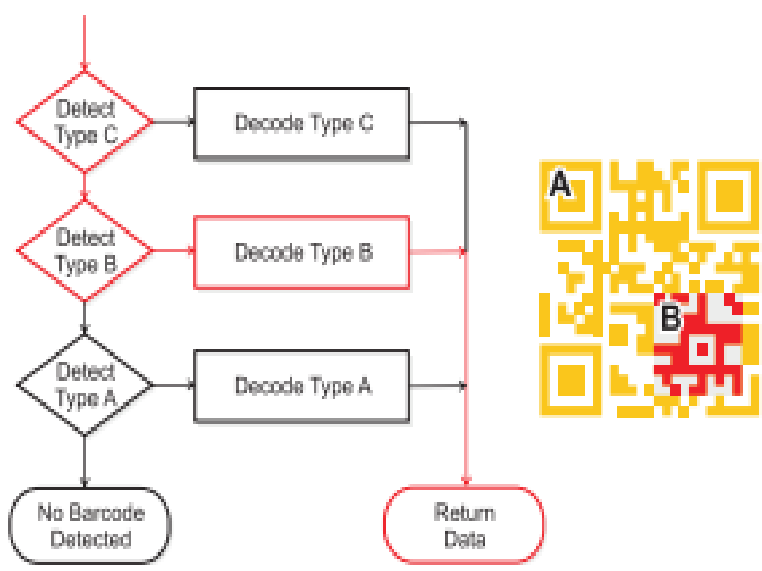

Figure 10. Attack: The inner barcode type is always detected before the outer one

For performing a phishing attacks as well as targeted exploits How embedding one barcode symbology into another is described In addition, for these barcode-inbarcode attacks on smartphones are susceptible popular 2D barcode reader applications is evaluate. Against this type of attack we discuss the mitigation techniques further.

\section{III.CONCLUSION}

In this paper a thorough survey of the existing barcode systems have been carried out to understand the encoding and barcoding techniques of existing barcodes. It can be analysed that as per the encoding techniques. The survey emphasizes on the various barcode techniques and checks whether the large data encoding into same dimension barcode is possible or not and can be concluded that a High capacity 2 dimensional barcode generation is essential for large data encoding into barcode.

\section{REFERENCES}

[1] SN. J. Woodland and B. Silver, "Classifying apparatus and method," U.S. Patent 2612 994, Oct. 7, 1952.

[2] Information Technology-Automatic Identification and Data Capture Techniques - QR Code 2005 Bar Code Symbology Specification, ISO/IEC 18004:2006, 2006.

[3] H. Kato and K. Tan, "Pervasive 2d barcodes for camera phone applications," Pervasive Comput., vol. 6, no. 4, pp. 76-85, Oct. 2007.

[4] X. Liu, D.Doermann, and H. Li, "Vcode-pervasive data transfer using video barcode," IEEE Trans. Multimedia, vol. 10, no. 3, pp. 361-371, Apr. 2008.

[5] S. D. Perli, N. Ahmed, and D. Katabi, "Pixnet: Interference-free wireless links using LCD-camera pairs," in Proc. MobiCom, 2010, pp. 137-148.

[6] J. Memeti, F. Santos, M. Waldburger, and B. Stiller, "Data transfer using a camera and a three dimensional code," Praxis der Informa tionsverarbeitung und Kommunikation, vol. 36, no. 1, pp. 31-37, 2013.

[7] C. Pei, Z. Zhang, and S. Zhang, "Softoc: Real-time projector-wallcamera communication system," in Proc. ICCE, Jan. 2013, pp. 100 101.

[8] S. Kuzdeba, A. M. Wyglinski, and B. Hombs, "Prototype implementation of a visual communication system employing video imagery," in Proc. CCNC, 2013, pp. 184-189.
[9] M. Mondal and J. Armstrong, "Impact of linear misalignment on a spatial OFDM based pixelated system," in Proc. 18th Asia-Pacific Conf. Commun., Oct. 2012, pp. 617-622.

[10] M. Mondal and J. Armstrong, "The effect of defocus blur on a spatial OFDM optical wireless communication system," in Proc. 14th Int. Conf. Transparent Opt. Netw., Jul. 2012, pp. 1-4.

[11] M. R. H. Mondal and J. Armstrong, "Analysis of the effect of vignetting on mimo optical wireless systems using spatial OFDM," J. Lightw. Technol., vol. 32, no. 5, pp. 922-929, Mar. 1, 2014.

[12] R. Gonzalez and R. Woods, Digital Image Processing, 3rd ed. Upper Saddle River, NJ, USA: Pearson Education, 2007.

[13] A. Sripad and D. Snyder, "A necessary and sufficient condition for quantization errors to be uniform and white," IEEE Trans. Acoust. Speech, Signal Process., vol. ASSP-25, no. 5, pp. 442-448, Oct. 1977.

[14] J. Proakis and M. Salehi, Digital Communications, 5th ed. New York, NY, USA: McGraw-Hill Education, 2007.

\section{BIOGRAPHIES}

Abhishek Mukhedkar Pursuing B.E. degree in Computer Science and Engineering from Dhole Patil College of Engineering, Pune, India.

Ninad Bansode Pursuing B.E. degree in Computer Science and Engineering from Dhole Patil College of Engineering, Pune, India.

Tousif Tamboli Pursuing B.E. degree in Computer Science and Engineering from Dhole Patil College of Engineering, Pune, India.

Dhanshree Holkar Pursuing B.E. degree in Computer Science and Engineering from Dhole Patil College of Engineering, Pune, India.

Amit Zore, Professor, Dhole Patil College of Engineering, Computer Science Department, Pune, India. 